\title{
LncRNA-TCONS_00034812 is upregulated in atherosclerosis and upregulates miR-21 through methylation in vascular smooth muscle cells
}

\author{
Dongsheng Lin ${ }^{1 \#}$, Xian Zhang ${ }^{2 \#}$, Chiyuan Zhang ${ }^{3}$, Qiao Jin ${ }^{4}$, Luping Jiang ${ }^{4}$ \\ ${ }^{1}$ Department of Cardiovascular Medicine, The First Hospital of Changsha, Changsha, China; ${ }^{2}$ Department of Cardiovascular Medicine, The People's \\ Hospital of Zhangjiajie, Zhangjiajie, China; ${ }^{3}$ Department of Cardiovascular Medicine, The Third Xiangya Hospital, Central South University, \\ Changsha, China; ${ }^{4}$ Department of Cardiovascular Medicine, University of South China Affiliated Changsha Central Hospital, Changsha, China \\ Contributions: (I) Conception and design: D Lin, X Zhang; (II) Administrative support: L Jiang; (III) Provision of study materials or patients: \\ X Zhang, C Zhang; (IV) Collection and assembly of data: D Lin, X Zhang, C Zhang; (V) Data analysis and interpretation: Q Jin, L Jiang; (VI) \\ Manuscript writing: All authors; (VII) Final approval of manuscript: All authors. \\ "These authors contributed equally to this work. \\ Correspondence to: Qiao Jin; Luping Jiang. University of South China Affiliated Changsha Central Hospital, 161 Shaoshan Rd., Yuhua District, \\ Changsha, China. Email: jinqiaonhdx@163.com; lukejlp@126.com.
}

Background: LncRNA-TCONS_00034812 is a critical player in the proliferation of aortic smooth muscle cells. It is known that artery injury plays an important role in atherosclerosis. However, the potential implication of LncRNA-TCONS_00034812 in atherosclerosis remains unclear. In this study, we collected artery specimens from patients with atherosclerosis and healthy controls to investigate the involvement of LncRNA-TCONS_00034812 in atherosclerosis.

Methods: Sixty patients with atherosclerosis and 60 controls, admitted at The First Hospital of Changsha (Changsha, China), between March 2017 and March 2019, were included. An artery biopsy was performed on all participants to obtain the artery specimens. Real-time quantitative PCR were performed to quantify the relative expression level of LncRNA-TCONS_00034812. Its role in atherosclerotic lesion was evaluated in (high fat diet) HFD-induced $\mathrm{ApoE}^{-/}$mice. Moreover, human aortic smooth muscle cells (HAOSMCs) was employed to study functional role of LncRNA-TCONS_00034812 overexpression and knockdown by methylation-specific PCR and cell proliferation assay.

Results: Overexpression of TCONS_00034812 resulted in miR-21 upregulation and a decrease of miR21 gene methylation. In contrast, silencing of TCONS_00034812 caused miR-21 downregulation and an increase of miR-21 gene methylation. Cell proliferation analysis indicated that the overexpression of TCONS_00034812 and miR-21 promoted cell proliferation, while silencing of TCONS_00034812 played an opposite role. Moreover, miR-21 overexpression weakened the effects of silencing TCONS_00034812 on cell proliferation.

Conclusions: In summary, LncRNA-TCONS_00034812 is upregulated in atherosclerotic samples, and its overexpression upregulates miR-21 through methylation in human aortic smooth muscle cells (HAOSMCs). Our study indicates that LncRNA-TCONS_00034812 could serve as a potential biomarker for diagnosis of atherosclerosis.

Keywords: LncRNA-TCONS_00034812; atherosclerosis; miR-21; methylation; aortic smooth muscle cells

Submitted Mar 24, 2021. Accepted for publication Jun 16, 2021.

doi: $10.21037 / \mathrm{atm}-21-2632$

View this article at: https://dx.doi.org/10.21037/atm-21-2632 


\section{Introduction}

Atherosclerosis is caused by the formation of plaques inside arteries, which are the result of an accumulation of cholesterol, fat, calcium, and other substances (1). Although atherosclerosis is not lethal, it increases the risk of other lethal diseases of the heart, such as coronary heart disease (2). At present, cholesterol-lowering therapy is widely used to treat early-stage atherosclerosis, which is reversible $(3,4)$. However, advanced atherosclerosis is not reversible, and cholesterol-lowering therapy is not effective for such cases $(3,4)$. Smoking, high blood pressure, and high cholesterol are the main risk factors for atherosclerosis (5). However, the molecular pathogenesis of this disease is still largely unknown (6), which limits the development of effective therapies.

DNA methylation is a crucial mechanism modulating genes in cell differentiation and pathological processes. Aberrant DNA methylation such as hypermethylation of DNA in the promoter region of tumor suppressor genes, is a well-studied epigenetic marker in various cancers (7). Methylated DNA immunoprecipitation (MeDIP), methylated CpG island amplification (MCA) as well as whole genome bisulfite sequencing have been used to profile methylation pattern changes in atherosclerotic samples (8-10). Microarray and RNA-seq have been successfully applied to profile the transcriptomic changes in the pathogenesis of atherosclerosis $(11,12)$. Many atherosclerosis-related genes have been found to be regulated through DNA methylation mechanism (13). Several recent studies demonstrated that patients with atherosclerosis display heightened level of DNA methylation in aortic samples and blood samples $(14,15)$, and the level of DNA methylation seems to correlate with atherosclerotic lesion progression (16). Altered methylation pattern modulates the expression changes of genes in inflammation, aging and oxidative stresses (13). For example, hypermethylation of the estrogen receptor gene $(\mathrm{ER} \alpha)$ is associated with aging and atherosclerosis, which may function to regulate smooth muscle cell proliferation (17). Genes associated with atherosclerosis, such as inflammatory markers and coagulation markers, can be used as biomarkers for cardiovascular risk prediction by the receiver operating characteristic (ROC) curve method (18).

Non-coding RNAs (ncRNAs) include miRNAs and long (>200 nt) ncRNAs (lncRNAs). NcRNAs participate in diverse pathological and physiological processes mainly by regulating gene expression (19). A large number of differentially expressed ncRNAs have been found in atherosclerosis and some of them are critical players in the regulation of vascular smooth muscle cells (VSMCs), which have important roles in the occurrence and development of atherosclerosis $(20,21)$. Atherosclerosis is initiated by endothelial dysfunction and neovascularization is a prominent feature of atherosclerotic lesions (22). The hyperplasia of the vasa vasorum in the artery wall is followed by neovascularization within the atherosclerotic plaque, and plaque hemorrhage is a feature of unstable plaques frequently happening in intimal neovessels (23). LncRNA-TCONS_00034812 was showed to regulate cell proliferation and apoptosis of pulmonary artery smooth muscle cells and its mechanism (24). However, its role in the neovascularization and plaque formation in atherosclerotic lesions is unclear. In addition, miR-21 targets tropomyosin 1 to promote the proliferation of VSMCs (25). In this study, we compared LncRNA-TCONS_00034812 and miR-21 expression in intima samples form healthy controls and atherosclerotic patients. We found both LncRNATCONS_00034812 and miR-21 expression was significantly upregulated in atherosclerotic samples, and atherosclerosis lesions were ameliorated by TCONS_00034812 knockdown in HFD-induced $\mathrm{ApoE}^{-/-}$atherosclerosis mouse model. The overexpression of LncRNA-TCONS_00034812 and miR21 also promoted the proliferation of human aortic smooth muscle cells (HAOSMCs) expression These results suggest that LncRNA-TCONS_00034812 and miR-21 may serve as a biomarker and therapeutic target for atherosclerosis. We present the following article in accordance with the MDAR reporting checklist (available at https://dx.doi.org/10.21037/ atm-21-2632).

\section{Methods}

\section{Participants}

Sixty patients with atherosclerosis and 60 controls, admitted at the Second Xiangya Hospital of Central South University between March 2017 and March 2019, were included. The clinical and demographic information for patients and controls is listed in Table 1. There were 33 males and 27 females among the patients with atherosclerosis with an age range of $23-35$ years (mean age $29.1 \pm 4.3$ years). The 60 controls included 33 males and 27 females with an age range of 23-34 years (mean age 29.0 4 .1 years). Histological analysis was performed on all patients to confirm atherosclerosis. All patients were at first-time diagnosis. Patients with other clinical complications or who had received other therapies in the previous 6 months were excluded. All procedures performed in this study involving human participants were in accordance with the Declaration of Helsinki (as revised in 2013). The 
Table 1 Clinical data of patients and controls

\begin{tabular}{lcc}
\hline Variable & Atherosclerosis $(\mathrm{n}=60)$ & Controls $(\mathrm{n}=60)$ \\
\hline Age & $29.1 \pm 4.3$ & $29.0 \pm 4.1$ \\
Gender & & \\
Male & 33 & 33 \\
Female & 37 & 37 \\
Smoking & 29 & 28 \\
hs-CRP (mg/L) & $4.67 \pm 0.23$ & $3.94 \pm 0.48$ \\
TG (mmol/L) & $1.72 \pm 1.11$ & $1.49 \pm 0.56$ \\
TC (mmol/L) & $4.19 \pm 0.38$ & $4.40 \pm 0.41$ \\
LDL-C (mmol/L) & $2.30 \pm 0.30$ & $2.27 \pm 0.33$ \\
HDL-C (mmol/L) & $1.23 \pm 0.40$ & $1.71 \pm 0.29$
\end{tabular}

hs-CRP, high-sensitivity C-reactive protein; TG, triglycerides; LDL-C, low-density lipoprotein cholesterol; HDL-C, high-density lipoprotein cholesterol.

study was approved by medical ethics board of the Second Xiangya Hospital of Central South University, Changsha, China (No.: PJ-KY-2020-128) and informed consent was taken from all the patients.

\section{Artery specimens}

An artery biopsy was performed on all participants to obtain the artery specimens. The artery biopsy was collected from the atherosclerosis-affected sites of the atherosclerosis patients. The 60 controls were diagnosed with suspected artery lesions, but lesions were subsequently excluded following biopsy. All specimens were from the temporal artery of the control subjects. Intima, the inner layer of the artery specimens, was separated and maintained in liquid nitrogen before use.

\section{Human aortic smootb muscle cells (HAOSMCs)}

HAOSMCs (354-05A, Sigma-Aldrich, , Saint Louis, Missouri, USA) were used in this study. The HAOSMCs were cultivated under the conditions described in the protocol from Sigma-Aldrich. HAOSMCs were harvested at passage 3 to 5 to perform the subsequent experiments.

\section{Cell transfections}

As a backbone, the pcDNA3.1 vector was used to construct the TCONS_00034812 (10) expression vector. MiR-21 mimic and negative control (NC) miR-21 were acquired from Sigma-Aldrich. HAOSMCs were collected at about $80 \%$ confluence and were counted. Lipofectamine 2000 (a kind of Invitrogen, Sigma-Aldrich, Saint Louis, Missouri, USA) was applied to transfect $10^{6}$ cells with $10 \mathrm{nM}$ vector, $40 \mathrm{nM}$ siRNA, or $40 \mathrm{nM}$ miRNA. The cells that were not transfected were adopted as the control (C) cells. The cells transfected by the empty vector, NC siRNA, or NC miRNA served as NC cells. Subsequent experiments were carried out $48 \mathrm{~h}$ after the transfection.

\section{RNA preparation and real-time quantitative PCR (RT- $q P C R)$}

The extraction of total RNAs from the intima samples and HAOSMCs was completed with Trizol (SigmaAldrich, Saint Louis, Missouri, USA). Eighty-five percent ethanol was applied to precipitate and wash the RNA samples, in order to harvest miRNAs. The extracted RNA samples were treated with a gDNA eraser (Takara), to digest the genomic DNAs. RNA concentrations were measured by a NanoDrop ${ }^{\mathrm{TM}} 2000$ spectrophotometer (Thermo Fisher, Waltham, MA, USA). Reverse transcription was performed with a QuantiTect Reverse Transcription Kit (QIAGEN, Düsseldorf, Germany) with total RNA as the template to complete the synthesis of the cDNA samples. PCR reactions were prepared with a KAPA SYBR ${ }^{\circledR}$ FAST qPCR Master Mix (Kapa Biosystems, Wilmington, Massachusetts, USA). The TCONS_00034812 expression was estimated with GAPDH as the endogenous control. The MiR-21 expression was calculated by using the All-in-One $\mathrm{e}^{\mathrm{TM}}$ miRNA qRT-PCR Reagent Kit (Genecopoeia, Rockville, Maryland, USA). The expression levels of miR-21 were normalized to the endogenous control miR-2 1 . The researchers repeated all experiments three times. The mean values were counted by the $2^{-\Delta \Delta C T}$ method to determine the fold changes of the gene expression levels.

\section{Animal protocol}

Male $\mathrm{ApoE}^{-/-}$mice were acquired from Beijing HFK Bioscience Co., Ltd. (Beijing, China). The 6-week-old mice weighed $28-32 \mathrm{~g}$. An atherogenic diet was fed to the $\mathrm{ApoE}^{-/-}$mice for 4 weeks to create an atherosclerosis model. The atherogenic diet included $2 \%$ cholate, $10 \%$ cholesterol, $10 \%$ lard, and $78 \%$ basal feed. After the establishment of 


\section{Page 4 of 10}

the model, the blood samples gathered via cardiac puncture were centrifuged at $1,500 \times \mathrm{g}$ at $4{ }^{\circ} \mathrm{C}$ for $10 \mathrm{~min}$ to acquire the serum. Subsequently, the mice were sacrificed. The aortic sinuses of the mice were cut out and stored in liquid nitrogen before use. Experiments were performed under a project license (No.: 20201115S0310211[308]) granted animal use ethics board of the Second Xiangya Hospital of Central South University, Changsha, China, in compliance with institutional Animal Care and Use Guidelines for the care and use of animals.

\section{Atherosclerotic lesion analysis by hematoxylin and eosin stainning}

After the dissection, the aortic sinuses were put in a $4 \%$ paraformaldehyde solution dehydrated with alcohol. Then the aortic sinuses were embedded in paraffin. Serial paraffin sections were made with a thickness of $5 \mu \mathrm{m}$, then stained with hematoxylin and eosin (H\&E) (Histoserv, Germantown, MD, USA). The necrotic core quantification and lesion areas were examined. An Olympus fluorescent microscope (DP80; Olympus Corp., Tokyo, Japan) was used to observe the morphological changes.

\section{Methylation-specific PCR (MSP)}

At $48 \mathrm{~h}$ after transfection, the Genomic DNA Isolation Kit (ab65358, Abcam, Cambridge, UK) was used to extract the genomic DNAs. DNA samples were alternated by the EZ DNA Methylation-Gold ${ }^{\mathrm{TM}}$ kit (ZYMO, Irvine, California, USA). Routine PCR reactions were performed with the Taq 2X Master Mix (NEB, Beijing, China) to detect gene methylation.

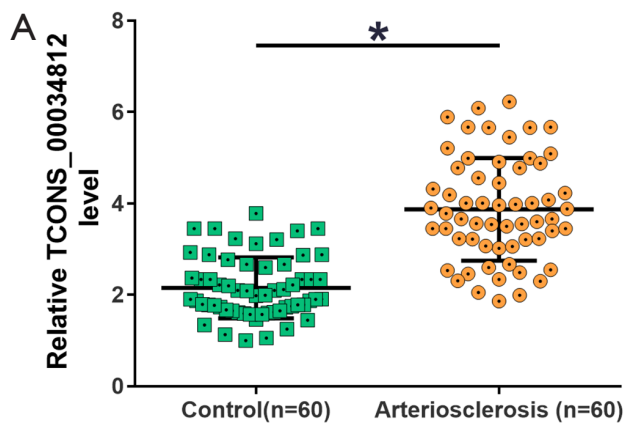

Lin et al. LncRNA-TCONS_00034812 and miR-21 in atherosclerosis

\section{Cell proliferation assay}

A CCK-8 assay (Dojindo, Japan) was performed to analyze the proliferation of HAOSMCs (harvested at $48 \mathrm{~h}$ posttransfection). The 3,000 cells in a $0.1-\mathrm{mL}$ cell culture medium were shifted to all the wells of a 96-well cell culture plate. At $4 \mathrm{~h}$ before cell collection, the cell cultivation was kept at $37^{\circ} \mathrm{C}$. Then CCK-8 solution was mixed into each well to achieve the ultimate concentration of $10 \%$. Cells were harvested every $24 \mathrm{~h}$ up to $96 \mathrm{~h}$. The OD value measurement was undertaken at $450 \mathrm{~nm}$ to report cell proliferation.

\section{Statistical analysis}

The above experiments were conducted in three independent biological replicates with the mean values calculated. The between-group differences were compared by unpaired $t$-tests, ANOVA (one-way), and the Tukey test. Correlations were analyzed by Pearson's correlation coefficient. A P value $<0.05$ was considered statistically significant.

\section{Results}

\section{TCONS_00034812 and miR-21 were upregulated in the intima samples from atherosclerosis patients}

The expression levels of TCONS_00034812 and miR-21 were measured from the intima samples of atherosclerosis patients $(n=60)$ and controls $(n=60)$. TCONS_00034812 expression was visibly increased in the atherosclerosis group compared with the control group (Figure $1 A, \mathrm{P}<0.05$ ), and MiR-21 expression was also markedly higher in the

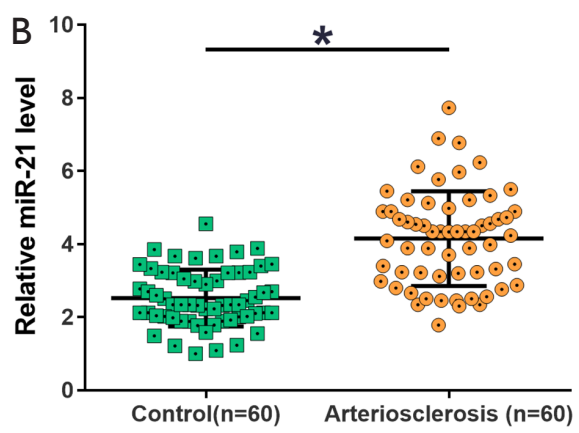

Figure 1 qPCR analysis revealed the upregulation of TCONS_00034812 and miR-21 were upregulated in intima samples from atherosclerosis patients. Expression levels of TCONS_00034812 (A) and miR-21 (B) in intima samples of atherosclerosis patients and controls were measured by performing RT-qPCR. *, P<0.05. RT-qPCR, real-time quantitative PCR. 

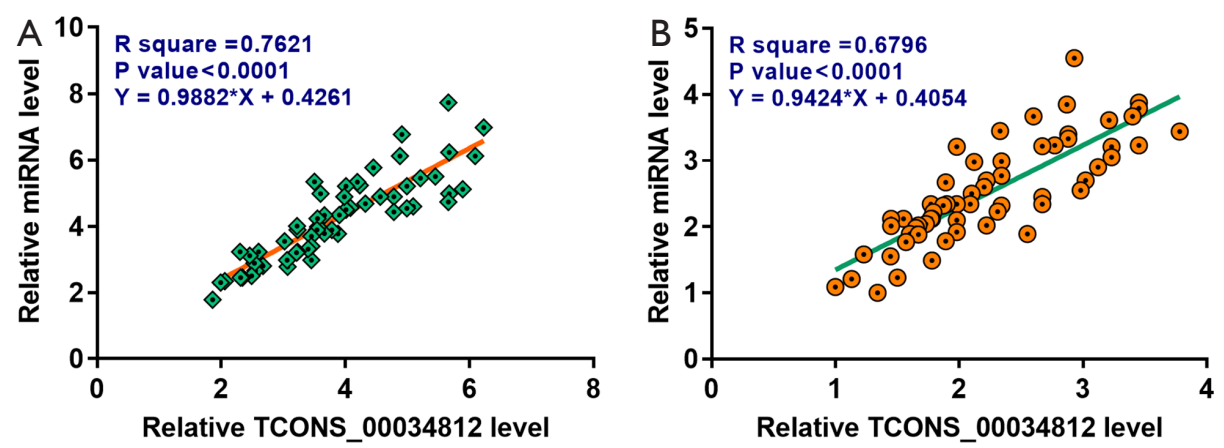

Figure 2 TCONS_00034812 and miR-21 were inversely associated across intima samples from atherosclerosis patients in a significant degree. The association between TCONS_00034812 expression and miR-21 expression across intima samples in atherosclerosis patients (A) and controls (B) were explored by performing Pearson's correlation coefficient.

atherosclerosis group than in the control group (Figure 1B, $\mathrm{P}<0.05)$.

\section{TCONS_00034812 and miR-21 were positively associated in the intima samples}

The association of the expression levels between TCONS_00034812 and miR-21 was explored by performing Pearson's correlation coefficient. Results demonstrated that TCONS_00034812 expression and miR21 expression were distinctly and positively associated across the intima samples of the atherosclerosis patients (Figure $2 A$ ). Furthermore, a positive and significant association between TCONS_00034812 and miR-21 was also reflected in the intima samples of the controls (Figure 2B).

\section{Atherosclerosis lesions were ameliorated by TCONS_00034812 knockdown in HFD-induced ApoE ${ }^{-/-}$ mice}

The atherosclerotic lesion areas were defined by $\mathrm{H} \& \mathrm{E}$ staining to further evaluate the effect of TCONS_00034812 knockdown on atherosclerotic development in $\mathrm{ApoE}^{-/-}$mice. Results showed that injection with si-TCONS_00034812 led to a more significant decrease in the atherosclerotic lesion area than in the si-NC group (Figure 3).

\section{TCONS_00034812 positively regulates the expression of miR-21 in HAOSMCs through methylation}

HAOSMCs were transfected by using the TCONS_00034812 expression vector, TCONS_00034812 siRNA or miR-21 mimic, to analyze the interaction between them. Transfections were confirmed at $48 \mathrm{~h}$ post-transfection by performing RT-qPCR (Figure $4 A, \mathrm{P}<0.05$ ). Compared with the NC and C groups, TCONS_00034812 overexpression caused the upregulation of miR-21 $(\mathrm{P}<0.05)$ and decreased the methylation of the miR-21 gene (Figure $4 B$ ). In contrast, silencing of TCONS_00034812 resulted in downregulation of miR-21 $(\mathrm{P}<0.05)$ and increased miR-21 gene methylation (Figure $4 C$ ), while miR-21 overexpression did not have any effect on TCONS_00034812 expression (Figure 4D).

\section{TCONS_00034812 regulated miR-21 to promote the proliferation of HAOSMCs}

The HAOSMC proliferation affected by the transfections was analyzed by a CCK- 8 assay. Overexpression of TCONS_00034812 and miR-21 promoted cell proliferation but silencing of TCONS_00034812 gave rise to a negative influence. In addition, miR-21 overexpression attenuated the consequences of silencing of TCONS_00034812 (Figure 5, $\mathrm{P}<0.05)$.

\section{Discussion}

The involvement of TCONS_00034812 in atherosclerosis was established in the current study. Our study showed that TCONS_00034812 was overexpressed in atherosclerosis and that it interacted with miR-21 through methylation to regulate the proliferation of HAOSMCs.

VSMCs play different roles at different stages of atherosclerosis (26-28). The increased proliferation rate of VSMCs can cause the formation of plaques, which then induce the development of atherosclerosis (26). In contrast, the aberrant proliferation of VSMCs in advanced 

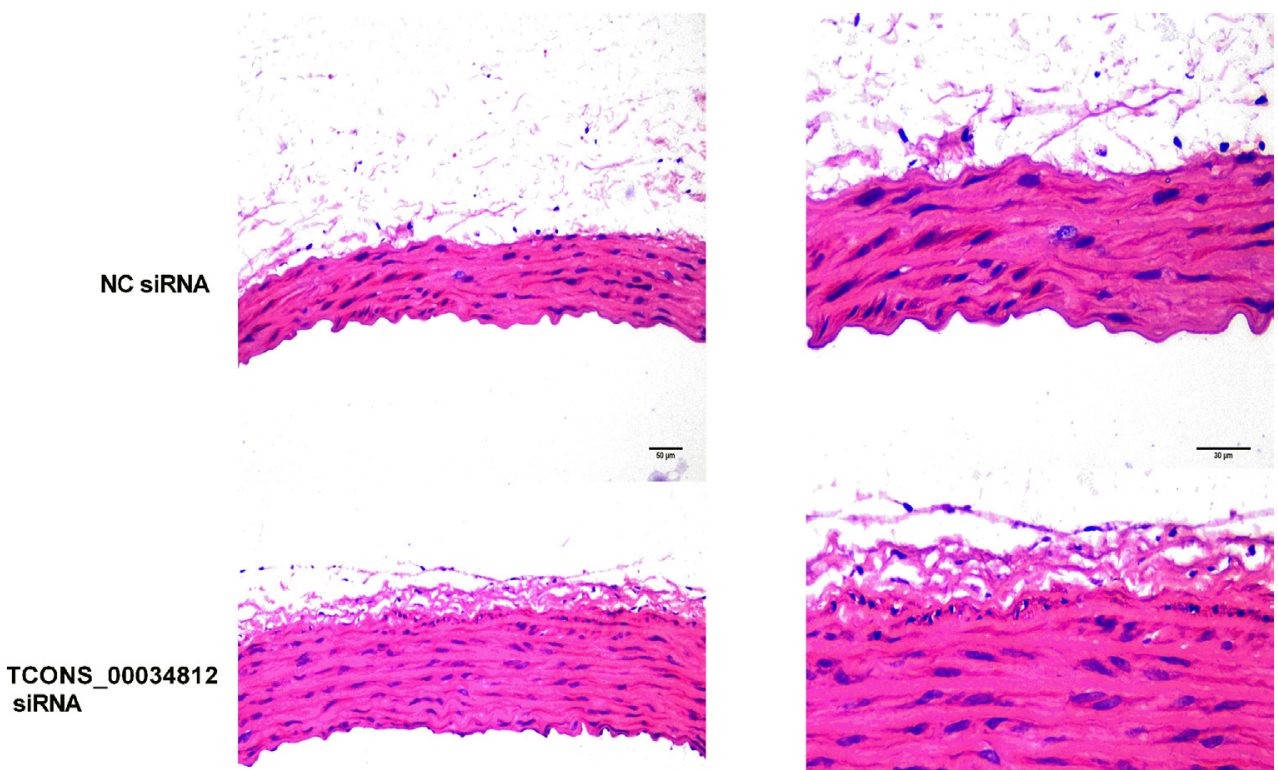

$200 \times$

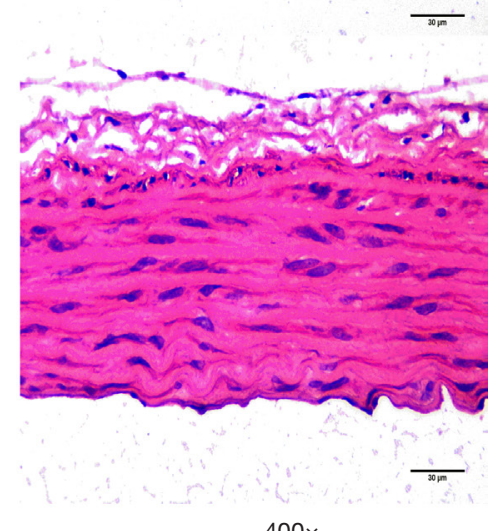

$400 x$

Figure 3 Detected by HE, atherosclerotic lesion was improved by TCONS_00034812 knockdown in HFD-induced ApoE ${ }^{-/-}$mice. Aortic sinus lesion area was showed to be smaller in si-TCONS_00034812-injected mice than in si-NC-injected mice, ${ }^{*}, \mathrm{P}<0.05$. NC siRNA, small interfering RNA control.

stage atherosclerosis plays a protective role by preventing fibrous cap rupture (26). A recent study reported that TCONS_00034812 was upregulated in a rat model of pulmonary artery hypertension, and its downregulation led to an increased proliferation rate of hypoxia in pulmonary artery smooth muscle cells (24). Interestingly, we also observed the upregulation of TCONS_00034812 in intima samples from atherosclerosis patients. We also observed the enhancing effects of TCONS_00034812 on the proliferation of HAOSMCs. The opposite results observed in this study might be due to the different types of VSMCs used in this study. More studies are necessary to investigate the influences of TCONS_00034812 in the proliferation of other types of VSMCs.

A growing body of evidence indicates that dysregulation of lncRNA expression may contribute to the pathogenesis of atherosclerosis and vascular injury (29). For example, lncRNA MIAT is found to be significantly upregulated in atherosclerotic lesions, resulting in a sponging effect on miR-149-5p and the inhibition of efferocytosis (30). lncRNA-H19 is proposed to augment angiogenesis and accelerates the development of atherosclerosis (31). Different lncRNAs are implicated in a wide spectrum of roles in atherosclerosis by recruiting leukocytes and immune cells, regulating lipid homeostasis and remodeling local vascular structures (29).

It has been reported that miR-21 is overexpressed in atherosclerosis and can target tropomyosin 1 to accelerate the proliferation of VSMCs (25). The same study also revealed the upregulation of miR-21 in atherosclerosis and its enhancing effects on the proliferation of VSMCs (25). Other research has reported that the methylation of miRNAs may be regulated by lncRNAs (32). Liu et al. showed that TCONS_00034812 could negatively regulate miR-21 gene methylation to positively adjust the expression of miR-21, thereby participating in the proliferation of HAOSMCs (24). However, there may also be other mechanisms involved, and this would require more research to confirm. It is known that TCONS_00034812 may regulate the proliferation and apoptosis of pulmonary artery smooth muscle cells by interacting with MAPK signaling, which plays a critical role in DNA hypomethylation (33). 

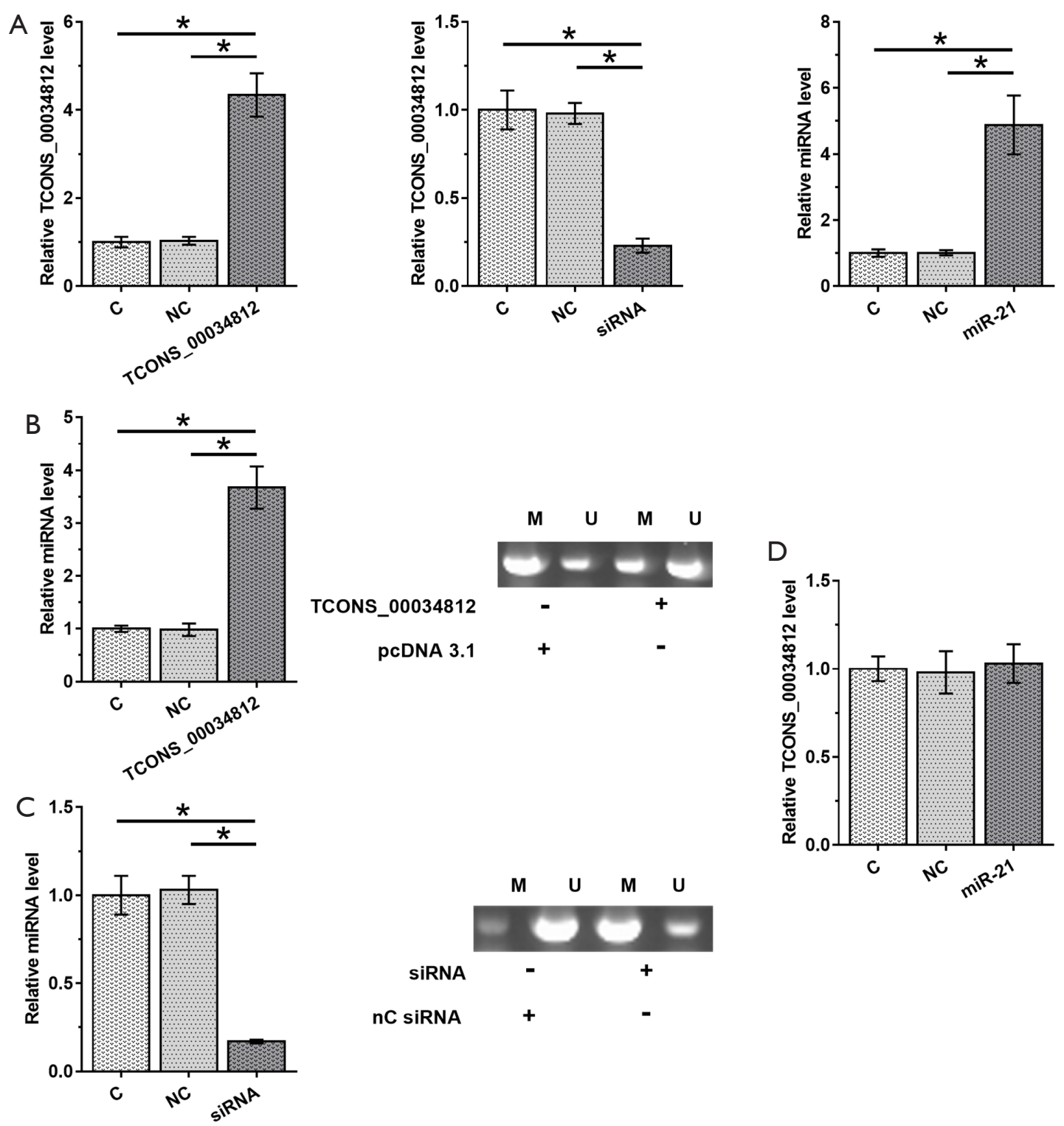

Figure 4 TCONS_00034812 positively regulate the expression of miR-21 in HAOSMCs through methylation. HAOSMCs were transfection with TCONS_00034812 expression vector, TCONS_00034812 siRNA or miR-21 mimic to analyze the interaction between them. Transfections were completed at $48 \mathrm{~h}$ after the transfection by proceeding RT-qPCR (A). The effects of overexpression of TCONS_00034812 (B) and silencing of TCONS_00034812 (C) on miR-21 expression and miR-21 gene methylation were evaluated by RT-qPCR and MSP. The effects of miR-21 overexpression on TCONS_00034812 were estimated by RT-qPCR (D). * P<0.05. HAOSMCs, human aortic smooth muscle cells; C, untreated control; NC, miRNA control; NC siRNA, small interfering RNA control.

Therefore, MAPK signaling may mediate the regulation of miR-21 methylation by TCONS_00034812.

Recent studies also highlighted a heightened level of DNA methylation in aortic samples and blood samples of patients with atherosclerosis $(13,14)$, and the level of DNA methylation seems to correlate with atherosclerotic lesion progression (15). Several factors could contribute to the development of atherosclerosis by modulating DNA methylation. For example, inflammation in cardiovascular diseases is reported to alter DNA methylation and proposed as a risk factor for atherosclerosis (34). Aging could lead to gene-specific hypermethylation, which may impact on genes 


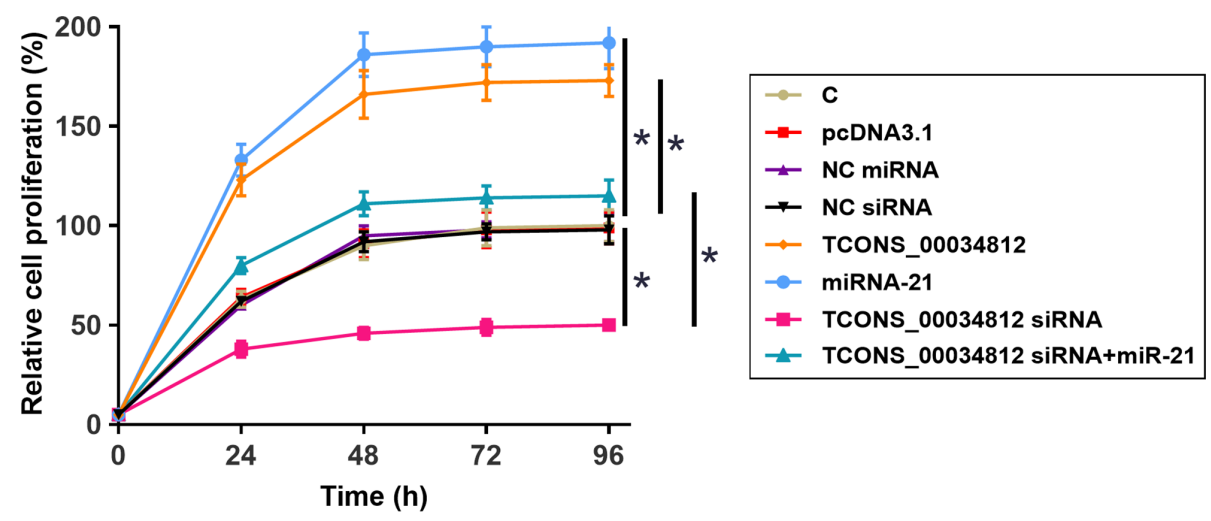

Figure 5 TCONS_00034812 regulated miR-21 to promote HAOSMCs proliferation. The effects of transfections on HAOSMCs proliferation were analyzed by CCK-8 assay. * $\mathrm{P}<0.05$. HAOSMCs, human aortic smooth muscle cells; C, untreated control; NC miRNA, miRNA control; NC siRNA, small interfering RNA control.

implicated in the atherosclerosis pathogenesis (17). On the other hand, chronic oxidative stress could also deregulate methylation signature of genes involved in the pathological development of atherosclerosis (35).

One limitation of this study is its small sample size. Also, our animal model experiments did not include an exploration of the interaction between TCONS_00034812 and miR21 in atherosclerosis. Our future studies will attempt to overcome these problems. Due to the increased incidence rate of atherosclerosis in young people in China, this study only included patients younger than 35 years. As it is known that atherosclerosis mainly affects older people, our future studies will include older patients to further explore the association between TCONS_00034812 and miR-21 in this disease. Finally, our control group consisted of people with suspected artery lesions, and therefore the temporal artery samples obtained were not truly normal arteries. This is due to the difficulty of obtaining normal arteries. Therefore, our conclusions remain to be further validated.

In conclusion, TCONS_00034812 is overexpressed in atherosclerosis and upregulates miR-21 through methylation in HAOSMCs, which affects their proliferation.

\section{Acknowledgments}

Funding: This study was supported by the National Natural Science Foundation of China (81373554).

\section{Footnote}

Reporting Checklist: The authors have completed the
MDAR reporting checklist. Available at https://dx.doi. org/10.21037/atm-21-2632

Data Sharing Statement: Available at https://dx.doi. org/10.21037/atm-21-2632

Conflicts of Interest: All authors have completed the ICMJE uniform disclosure form (available at https://dx.doi. org/10.21037/atm-21-2632). The authors have no conflicts of interest to declare.

Ethical Statement: The authors are accountable for all aspects of the work in ensuring that questions related to the accuracy or integrity of any part of the work are appropriately investigated and resolved. All procedures for human sample collection performed in this study involving human participants were in accordance with the Declaration of Helsinki (as revised in 2013). The study was approved by ethics board of the Second Xiangya Hospital of Central South University, Changsha, China (No.: PJKY-2020-128) and informed consent was taken from all the patients. All animal experiments were performed under a project license (No.: 20201115S0310211[308]) granted animal use ethics board of the Second Xiangya Hospital of Central South University, Changsha, China, in compliance with institutional Animal Care and Use Guidelines for the care and use of animals.

Open Access Statement: This is an Open Access article distributed in accordance with the Creative Commons Attribution-NonCommercial-NoDerivs 4.0 International 
License (CC BY-NC-ND 4.0), which permits the noncommercial replication and distribution of the article with the strict proviso that no changes or edits are made and the original work is properly cited (including links to both the formal publication through the relevant DOI and the license). See: https://creativecommons.org/licenses/by-nc-nd/4.0/.

\section{References}

1. Tarkin JM, Dweck MR, Evans NR, et al. Imaging Atherosclerosis. Circ Res 2016;118:750-69.

2. McClelland RL, Jorgensen NW, Budoff M, et al. 10-Year Coronary Heart Disease Risk Prediction Using Coronary Artery Calcium and Traditional Risk Factors: Derivation in the MESA (Multi-Ethnic Study of Atherosclerosis) With Validation in the HNR (Heinz Nixdorf Recall) Study and the DHS (Dallas Heart Study). J Am Coll Cardiol 2015;66:1643-53.

3. Writing Committee; Lloyd-Jones DM, Morris PB, et al. 2016 ACC Expert Consensus Decision Pathway on the Role of Non-Statin Therapies for LDL-Cholesterol Lowering in the Management of Atherosclerotic Cardiovascular Disease Risk: A Report of the American College of Cardiology Task Force on Clinical Expert Consensus Documents. J Am Coll Cardiol 2016;68:92-125.

4. Guo Y. What do we learn from the 2016 American College of Cardiology expert consensus decision pathway on nonstatin therapies for low-density lipoproteincholesterol lowering in the management of atherosclerotic cardiovascular disease risk? Cardiology Plus 2016;1:5.

5. Rafieian-Kopaei M, Setorki $M$, Doudi M, et al. Atherosclerosis: process, indicators, risk factors and new hopes. Int J Prev Med 2014;5:927-46.

6. Weber C, Noels H. Atherosclerosis: current pathogenesis and therapeutic options. Nat Med 2011;17:1410-22.

7. Sharma S, Kelly TK, Jones PA. Epigenetics in cancer. Carcinogenesis 2010;31:27-36.

8. Zaina S, Heyn H, Carmona FJ, et al. DNA methylation map of human atherosclerosis. Circ Cardiovasc Genet 2014;7:692-700.

9. Kim JY, Choi BG, Jelinek J, et al. Promoter methylation changes in ALOX12 and AIRE1: novel epigenetic markers for atherosclerosis. Clin Epigenetics 2020;12:66.

10. Xue Y, Guo Y, Luo S, et al. Aberrantly MethylatedDifferentially Expressed Genes Identify Novel Atherosclerosis Risk Subtypes. Front Genet 2020;11:569572.

11. Perisic L, Hedin E, Razuvaev A, et al. Profiling of atherosclerotic lesions by gene and tissue microarrays reveals PCSK6 as a novel protease in unstable carotid atherosclerosis. Arterioscler Thromb Vasc Biol 2013;33:2432-43.

12. Williams JW, Winkels H, Durant CP, et al. Single Cell RNA Sequencing in Atherosclerosis Research. Circ Res 2020;126:1112-26.

13. Tabaei S, Tabaee SS. DNA methylation abnormalities in atherosclerosis. Artif Cells Nanomed Biotechnol 2019;47:2031-41.

14. Chen WD, Song T, Cao QH, et al. Atherosclerosis prediction by microarray-based DNA methylation analysis. Exp Ther Med 2020;20:2863-9.

15. Jiang D, Sun M, You L, et al. DNA methylation and hydroxymethylation are associated with the degree of coronary atherosclerosis in elderly patients with coronary heart disease. Life Sci 2019;224:241-8.

16. Valencia-Morales Mdel P, Zaina S, Heyn H, et al. The DNA methylation drift of the atherosclerotic aorta increases with lesion progression. BMC Med Genomics 2015;8:7.

17. Post WS, Goldschmidt-Clermont PJ, Wilhide CC, et al. Methylation of the estrogen receptor gene is associated with aging and atherosclerosis in the cardiovascular system. Cardiovasc Res 1999;43:985-91.

18. Brown TM, Bittner V. Biomarkers of atherosclerosis: clinical applications. Curr Cardiol Rep 2008;10:497-504.

19. Mattick JS, Makunin IV. Non-coding RNA. Hum Mol Genet 2006;15 Spec No 1:R17-29.

20. Aryal B, Rotllan N, Fernández-Hernando C. Noncoding RNAs and atherosclerosis. Curr Atheroscler Rep 2014;16:407.

21. Zhou T, Ding JW, Wang XA, et al. Long noncoding RNAs and atherosclerosis. Atherosclerosis 2016;248:51-61.

22. Barrabés JA, Mirabet M. Neovascularization in atherosclerotic lesions: homeostatic response or mechanism of progression of the disease?. Rev Esp Cardiol 2003;56:947-8.

23. Camaré C, Pucelle $M$, Nègre-Salvayre A, et al. Angiogenesis in the atherosclerotic plaque. Redox Biol 2017;12:18-34.

24. Liu Y, Sun Z, Zhu J, et al. LncRNA-TCONS_00034812 in cell proliferation and apoptosis of pulmonary artery smooth muscle cells and its mechanism. J Cell Physiol 2018;233:4801-14.

25. Wang M, Li W, Chang GQ, et al. MicroRNA-21 regulates vascular smooth muscle cell function via targeting tropomyosin 1 in arteriosclerosis obliterans 


\section{Page 10 of 10}

of lower extremities. Arterioscler Thromb Vasc Biol 2011;31:2044-53.

26. Bennett MR, Sinha S, Owens GK. Vascular Smooth Muscle Cells in Atherosclerosis. Circ Res 2016;118:692-702.

27. Durham AL, Speer MY, Scatena M, et al. Role of smooth muscle cells in vascular calcification: implications in atherosclerosis and arterial stiffness. Cardiovasc Res 2018;114:590-600.

28. Grootaert MOJ, Moulis M, Roth L, et al. Vascular smooth muscle cell death, autophagy and senescence in atherosclerosis. Cardiovasc Res 2018;114:622-34.

29. Pierce JB, Feinberg MW. Long Noncoding RNAs in Atherosclerosis and Vascular Injury: Pathobiology, Biomarkers, and Targets for Therapy. Arterioscler Thromb Vasc Biol 2020;40:2002-17.

30. Ye ZM, Yang S, Xia YP, et al. LncRNA MIAT sponges miR-149-5p to inhibit efferocytosis in advanced atherosclerosis through CD47 upregulation. Cell Death

Cite this article as: Lin D, Zhang X, Zhang C, Jin Q, Jiang L. LncRNA-TCONS_00034812 is upregulated in atherosclerosis and upregulates miR-2 1 through methylation in vascular smooth muscle cells. Ann Transl Med 2021;9(12):1005. doi: 10.21037/atm-21-2632

\section{Lin et al. LncRNA-TCONS_00034812 and miR-21 in atherosclerosis}

Dis 2019;10:138.

31. Shi X, Wei YT, Li H, et al. Long non-coding RNA H19 in atherosclerosis: what role? Mol Med 2020;26:72.

32. Liu HT, Fang L, Cheng YX, et al. LncRNA PVT1 regulates prostate cancer cell growth by inducing the methylation of miR-146a. Cancer Med 2016;5:3512-9.

33. Deng C, Yang J, Scott J, et al. Role of the ras-MAPK signaling pathway in the DNA methyltransferase response to DNA hypomethylation. Biol Chem 1998;379:1113-20.

34. Wierda RJ, Geutskens SB, Jukema JW, et al. Epigenetics in atherosclerosis and inflammation. J Cell Mol Med 2010;14:1225-40.

35. Kobayashi S, Inoue N, Azumi H, et al. Expressional changes of the vascular antioxidant system in atherosclerotic coronary arteries. J Atheroscler Thromb 2002;9:184-90.

(English Language Editor: D. Fitzgerald) 